${ }^{2}$ Chiu, H.-Y., Quasi-Stellar Sources and Gravitational Collapse (edit. by Robinson, I., Schild, A., and Schucking, E. L. (University of Chicago Press, Chicago, 1965).

Fowler, W. A., and Hoyle, F., Nucleosynthesis in Massive Stars and Supernovae (University of Chicago Press, Chicago, 1964).

${ }^{4}$ Rakavy, G., Shaviv, G., and Zimamon, Z., Astrophys. J., 150, 131 (1967). Arnett, W. D., Supernoxae Conf., New York, November 1967 (in the press).

${ }^{5}$ Colgate, S. A., and White, R., Astrophys. J., 143, 626 (1966).

${ }^{6}$ Arnett, W. D., Astrophys. J., 153, 341 (1968).

? Weigert, A., Zeit. Astrophys., 64, 395 (1966). Murai, T., Sugimoto, D. Hoshi, R., and Hayashi, C., Prog. Theor. Phys., 39, 619 (1968); Beaudet, Hoshi, R., ind Hayashi, C., Prog. Theor. Phys., 39, 61
G., and Salpeter, E. E., Astrophys. J., 155, 203 (1969).

${ }^{8}$ Arnett, W. D., Nature, 219, 1344 (1968); Rose, W. K., Astrophys, J., 155, 491 (1969).

${ }^{9}$ Chandrasekhar, S., and Tooper, R. F., Astrophys. J., 139, 1396 (1964).

${ }^{10}$ Minkowski, R., Nebulae and Interstellar Matter, Stars and Stellar Systems (edit. by Aller, L. H., and McLaughlin, D. B.), $\mathbf{8}$ (University of Chicago Press, Chicago, 1968). Burn, B. J., Mon. Not. Roy. Astro. Soc., 133, 67 (1966).

11 Trimble, V., Astron. J., '73, 535 (1968).

12 Blaauw, A., Bull. Astron. Inst. Neth., 15, 265 (1961).

\section{Hydrogen Atoms in Interstellar Grains}

Pнотометеr measurements of stellar fluxes in the vacuum ultraviolet ${ }^{1-3}$ have shown that there exists a general flux deficiency below $1215 \AA$ (Lyman- $\alpha$ ). This observation is supported by the photographic spectra of Carruthers ${ }^{4}$, who suggests that this deficiency is not a result of line blanketing in the atmospheres of the stars studied, but is caused by absorption in some constituent of the interstellar medium. I examine here the possibility that the source of this absorption may be hydrogen atoms trapped at or near the surface of interstellar grains. The influence of a crystal environment on the Lyman- $\alpha$ transition of atomic hydrogen was investigated.

Detailed calculation of perturbations to the energy levels of an impurity atom trapped in a solid usually requires knowledge of a number of parameters which are not available for interstellar grains ${ }^{5,6}$. A more general approach is therefore necessary. I have used the continuous dielectric model of Coulson et al. ${ }^{7,8}$, which has the advantage that a reasonable estimate of the shift of an atomic transition of a trapped impurity can be obtained from one parameter: the dielectric constant.

Following Jortner and Coulson ${ }^{8}$ it is assumed that a trapped hydrogen atom resides in the centre of a spherical

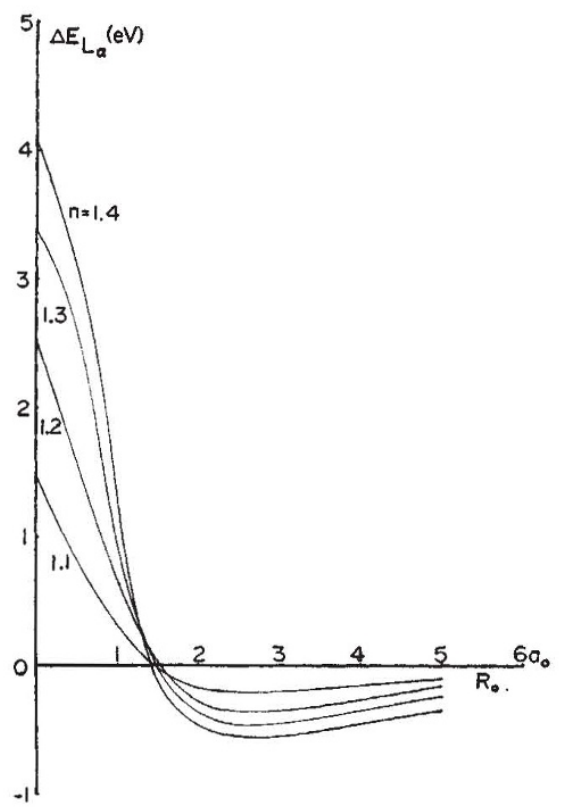

Fig. 1. $\Delta E_{L a}$ versus $R_{0}$ for various values of $n$ the refractive index. $R_{0}$ is in atomic units. When $\Delta E_{L \alpha}$ is negative Lyman- $\alpha$ is shifted to shorter wavelengths. cavity of radius $R_{o}$ in a continuous dielectric medium. This medium is characterized by an optical dielectric constant $D_{o}$ and a static dielectric constant $D_{\delta}$. The shift of the ground state, $\Delta E(1 s)$, can be found from

$$
\begin{gathered}
\lambda=1-\alpha_{s}\left[\frac{11}{16}+\frac{11 X}{4}+\frac{7 X^{2}}{2}+2 X^{3}\right] \exp (-4 X) \\
E(\lambda)=\frac{\lambda^{2}}{2}-\lambda+\frac{\alpha_{s}}{R_{0}} \\
{\left[(1+X) \exp (-2 X)-\left(1+\frac{21 X}{8}+\frac{5 X^{2}}{2}+X^{3}\right) \exp (-4 X)\right]}
\end{gathered}
$$

and

$$
\Delta E(1 s)=E_{f}(1 s)-E(\lambda)
$$

where $\alpha_{s}=1-1 / D_{s}, X=\lambda R_{0}$ and $E_{f}(1 s)=$ the ground state energy of a free hydrogen atom. The shift of the $2 p$ level is

$$
\Delta E(2 p)=\int_{0}^{R_{0}}\left|\psi_{2} p\right|^{2} V_{p}\left(R_{0}\right) \mathrm{d} \tau+\int_{R_{0}}^{\infty}\left|\psi_{2} p\right|^{2} \mathrm{~V}_{p}(r) \mathrm{d} \tau
$$

with

$$
\begin{aligned}
V_{p}(r)=\left(\alpha_{\varepsilon}-\right. & \left.\alpha_{0}\right)\left(\frac{1+\lambda r}{r}\right) \exp (-2 \lambda r) \\
& +\frac{\alpha_{0}}{r}\left(1+\frac{3 v r}{2}+\nu^{2} r^{2}+\frac{\nu^{3} r^{3}}{3}\right) \exp (-2 v r)
\end{aligned}
$$

and

$$
\psi_{2} p(r)=\left(\frac{v^{5}}{3 \pi}\right)^{1 / 2} r \exp (-v r) \text { with } \nu=\frac{1}{2}
$$

and $\alpha_{0}=\left(1-1 / D_{o}\right)$. The shift cf Lyman- $\alpha$ is

$$
\Delta E_{L a}=\Delta E(1 s)-\Delta E(2 p)
$$

This shift is plotted in Fig. 1 for $D_{s}=D_{0}=n^{2}$, where $n$ is the (real) refractive index. As a check on the validity of these approximations the shift of Lyman- $\alpha$ in solid argon is found to be $\simeq+0.40 \mathrm{eV}$ when $R_{o}$ is taken as one-half the nearest neighbour separation ${ }^{8}$. The experimental shift is $+0 \cdot 36 \mathrm{eV}$ (ref. 9). This agreement indicates that long range forces dominate the interaction between a trapped hydrogen atom and a matrix of neutral species.

This calculation shows that, because $R_{0}$ for most materials lies in the range 2-5a, the Lyman- $\alpha$ transition of a hydrogen atom trapped in a material of refractive index $1 \cdot 1$ or greater would be shifted to higher energies. If $n=1.3$ and $R_{0}$ is between 3 and $4 a_{0}$, Lyman- $\alpha$ is shifted by about $+0.4 \mathrm{eV}$ regardless of the detailed structure of the material in the grain. Hydrogen atoms in interstellar grains would then give rise to a broad absorption feature centred about $1170 \AA$. It is interesting that the flux measurements of Opal et al. ${ }^{3}$ seem to exhibit a minimum at $\simeq 1180 \AA$. On the basis of this calculation the dip in photon flux below $1215 \AA$ may be a result of absorption by hydrogen atoms trapped at or near the surface of interstellar grains.

This research was supported by the National Research. Council.

\section{W. W. Duley}

Centre for Research in Experimental Space Science, Department of Physics,

York University,

Toronto, Canada.

Received February 3, 1969.

${ }^{1}$ Chubb, T. A., and Bryam, E. T., Astrophys. J., 138, 617 (1963).

'Bryam, E. T., Chubb, T. A., and Werner, N. W., Ann. d'Astrophys., 28, 594 (1965).

${ }^{3}$ Opal, C. B., Moos, H. W., Fastie, W. G., Bottema, M., and Henry, R. C., Astrophys. J., 153, L179 (1968).

4 Carruthers, G. R., Astrophys. J., 151, 269 (1968).

${ }^{5}$ Brith, M., and Schnepp, O., J. Chem. Phys., 39, 2714 (1963).

' Keil, T. H., and Gold, A., Phys. Rev., 136, A252 (1964).

${ }^{7}$ Coulson, C. A., Proc. Roy. Soe., A, 255, 69 (1960).

${ }^{8}$ Jortner, J., and Coulson, C. A., Mol. Phy8., 4, 451 (1961).

'Baldini, G., Phys. Rev., 186, A248 (1964). 